\title{
Error Estimates for a Finite Element Approximation of a Minimal Surface
}

\author{
By Claes Johnson and Vidar Thomée
}

\begin{abstract}
A finite element approximation of the minimal surface problem for a strictly convex bounded plane domain $\Omega$ is considered. The approximating functions are continuous and piecewise linear on a triangulation of $\Omega$. Error estimates of the form $O(h)$ in the $H^{1}$ norm and $O\left(h^{2}\right)$ in the $L_{p}$-norm $(p<2)$ are proved, where $h$ denotes the maximal side in the triangulation.
\end{abstract}

1. Introduction. Let $\Omega$ be a strictly convex bounded domain in the plane $R^{2}$ with smooth (two times continuously differentiable, say) boundary $\Gamma$, and let $\varphi$ be a given function defined on $\Gamma$. Consider the following minimal surface problem: Find a function $u$ which minimizes the integral

$$
\int_{\Omega} \sqrt{1+|\nabla v|^{2}} d x, \quad \nabla v=\operatorname{grad} v
$$

over all Lipschitz functions $v$ in $\Omega$ such that $v=\varphi$ on $\Gamma$. It is known (see, e.g., [2, Theorem 4.2.1]) that if $\varphi$ is the restriction to $\Gamma$ of a function in the Sobolev space $W_{q}^{2}(\Omega)$ for some $q>2$, and if $\varphi$ satisfies the bounded slope condition (see [2]), then there is a unique minimizing function $u \in W_{q}^{2}(\Omega)$.

For the purpose of the approximate solution of this problem, for each $h$ with $0<h<1$, let $T_{h}=\left\{T_{j}\right\}$ be a finite collection of closed triangles $T_{j}$ such that $\Omega \subset$ $\bigcup_{j} T_{j}$, and such that any $T_{j}$ with $T_{j} \cap \Omega \neq \varnothing$ is either contained in $\bar{\Omega}$ or has two vertices on $\Gamma$. It is also assumed that the triangles have disjoint interiors, that no vertex of any triangle is on the interior of an edge of another triangle, and that there is a constant $c$, with $0<c<1$ independent of $h$, such that the edges of the triangles have length between $c h$ and $h$, and all angles of the triangles are bounded below by $c$. Denoting the union of the triangles contained in $\bar{\Omega}$ by $\Omega_{h}$, we let $S_{h}$ be the set of continuous functions defined on $\Omega_{n}$ which are linear on each $T_{j}$ and assume the same values as $\varphi$ on the vertices of the triangulation on $\Gamma$. Consider now the following finite element method for the approximate solution of the given problem: Find a function $u_{n}$ which minimizes the integral $\int_{\Omega_{n}} \sqrt{1+\left|\nabla v_{n}\right|^{2}} d x$ over all functions $v_{n} \in S_{n}$. To see that there exists a unique minimizing function $u_{h}$, we notice that the function

$$
f(y)=\sqrt{1+|y|^{2}}, \quad y=\left(y_{1}, y_{2}\right) \in R^{2}, \quad|y|^{2}=y_{1}^{2}+y_{2}^{2},
$$


is strictly convex since, with $f_{, i j}=\partial^{2} f / \partial y_{i} \partial y_{j}$,

$$
\begin{aligned}
f_{, i j}(y) \xi_{i} \xi_{j} & =\left(1+|y|^{2}\right)^{-3 / 2}\left[\left(1+y_{2}^{2}\right) \xi_{1}^{2}-2 y_{1} y_{2} \xi_{1} \xi_{2}+\left(1+y_{1}^{2}\right) \xi_{2}^{2}\right] \\
& \geqslant\left(1+|y|^{2}\right)^{-3 / 2}|\xi|^{2} \quad \text { for } \xi \in R^{2}
\end{aligned}
$$

Here and below, we use the summation convention; repetition of an index $i$ indicates summation over $i=1,2$. Since $f$ is strictly convex, the mapping $F: v_{h} \rightarrow \int_{\Omega_{h}} f\left(\nabla v_{h}\right) d x$, $v_{h} \in S_{h}$, is also strictly convex. Furthermore, it is clear that $F\left(v_{h}\right)$ tends to infinity with $\max _{\Omega_{h}}\left|v_{h}\right|$. Since $F$ is continuous and $S_{h}$ is finite dimensional, it then follows easily that there exists a unique minimizing function $u_{n}$.

In this note, we shall prove some convergence estimates for the finite element method described above. In order to express our results, we introduce for $k$ an integer, $1 \leqslant p \leqslant \infty$, the following (semi) norms:

$$
|v|_{k, p}=\left(\sum_{|\alpha|=k} \int_{\Omega}\left|D^{\alpha} v\right|^{p} d x\right)^{1 / p}, \quad\|v\|_{k, p}=\left(\sum_{j \leqslant k}|v|_{k, p}^{p}\right)^{1 / p},
$$

with the usual modification if $p=\infty$. We shall also need corresponding norms with $\Omega$ replaced by $\Omega_{h}$, and we shall then use the notation $|\cdot|_{k, p, h}$ and $\|\cdot\|_{k, p, n}$. We introduce the Sobolev space $W_{p}^{k}(\Omega)$, the closure of $C^{\infty}(\Omega)$ in the norm $\|\cdot\|_{k, p}$, and the Sobolev space $W_{1}^{k}(\Gamma)$, the closure of $C^{\infty}(\Gamma)$ in the norm

$$
\|v\|_{k, 1, \Gamma}=\sum_{j \leqslant k} \int_{\Gamma}\left|\frac{d^{j} v}{d s^{j}}\right| d s
$$

where $d / d s$ denotes differentiation with respect to arc length. If $k=0$, we omit this index. For example, $\|\cdot\|_{p, h}$ will thus denote the $L_{p}$-norm over $\Omega_{h}$.

We can now state our convergence results.

THEOREM 1. Let $u \in W_{2}^{2}(\Omega) \cap W_{\infty}^{1}(\Omega)$. Then, there is a constant $C$ such that for $0<h<1$,

$$
\left|u-u_{h}\right|_{1,2, h} \leqslant C h .
$$

TheOREM 2. Let $u \in W_{q}^{2}(\Omega)$ for some $q>2$ and $\varphi \in W_{1}^{2}(\Gamma)$. Then, for any $p$ with $1 \leqslant p<2$, there is a constant $C$ such that, for $0<h<1$,

$$
\left\|u-u_{h}\right\|_{p, h} \leqslant C h^{2}
$$

The proofs of these estimates are given in Sections 2 and 3, respectively. For linear equations, such results are well known (cf., e.g., [3]); the latter then holds for $p=q=2$.

2. Proof of Theorem 1. Since $u_{n}$ minimizes the functional $F$ over $S_{h}$, we find, taking first variations, denoting by $v_{, i}$ the derivative of $v$ with respect to the $i$ th variable, that 


$$
\int_{\Omega_{n}} f_{, i}\left(\nabla u_{n}\right) \chi_{, i} d x=\int_{\Omega_{n}} \frac{\nabla u_{n} \nabla \chi}{\sqrt{1+\left|\nabla u_{n}\right|^{2}}} d x=0 \quad \text { for } \chi \in \stackrel{\circ}{S}_{n}
$$

where $\stackrel{\circ}{S}_{n}$ is the set of continuous functions defined on $\Omega_{n}$ which are linear on each $T_{j}$ and vanish on the boundary of $\Omega_{n}$. Let us extend the functions in $\stackrel{\circ}{S}_{h}$ to be zero outside $\Omega_{n}$. Then the functions in $\stackrel{\circ}{h}_{n}$ are Lipschitz continuous and vanish on the boundary of $\Omega$ so that, taking first variations in the continuous problem,

$$
\int_{\Omega} f_{, i}(\nabla u) \chi_{, i} d x=\int_{\Omega_{n}} \frac{\nabla u \nabla \chi}{\sqrt{1+|\nabla u|^{2}}} d x=0 \text { for } \chi \in \stackrel{\circ}{S}_{n} .
$$

Theorem 1 will be an obvious consequence of Lemmas 1 and 2 below.

Lemma 1. Let $u \in W_{2}^{2}(\Omega) \cap W_{\infty}^{1}(\Omega)$. Then, there is a constant $C$ such that for $0<h<1$,

$$
\left(\int_{\Omega_{n}} \frac{\left|\nabla u-\nabla u_{n}\right|^{2}}{\sqrt{1+\left|\nabla u_{n}\right|^{2}}} d x\right)^{1 / 2} \leqslant C h
$$

Proof. Let $w_{n}$ be any function in $S_{n}$, and set $\chi=w_{n}-u_{n}$. Then $\chi \in \stackrel{\circ}{S}_{n}$ and, using (2.1) and (2.2), we find

$$
\begin{aligned}
A^{2} & =\int_{\Omega_{n}} \frac{\left|\nabla u-\nabla u_{n}\right|^{2}}{\sqrt{1+\left|\nabla u_{n}\right|^{2}}} d x \\
& =\int_{\Omega_{n}} \frac{\left(\nabla u-\nabla u_{n}\right) \nabla x}{\sqrt{1+\left|\nabla u_{n}\right|^{2}}} d x+\int_{\Omega_{h}} \frac{\left(\nabla u-\nabla u_{n}\right)\left(\nabla u-\nabla w_{h}\right)}{\sqrt{1+\left|\nabla u_{n}\right|^{2}}} d x \\
& =\int_{\Omega_{n}} \nabla u \nabla x\left(\frac{1}{\sqrt{1+\left|\nabla u_{n}\right|^{2}}}-\frac{1}{\sqrt{1+|\nabla u|^{2}}}\right) d x+\int_{\Omega_{n}} \frac{\left(\nabla u-\nabla u_{n}\right)\left(\nabla u-\nabla w_{h}\right)}{\sqrt{1+\left|\nabla u_{n}\right|^{2}}} d x \\
& =D_{1}+D_{2} .
\end{aligned}
$$

For the second term, we find by Cauchy's inequality, $\left|D_{2}\right| \leqslant A\left|u-w_{n}\right|_{1,2, n}$. For the first term, we obtain with $\gamma=\max _{\bar{\Omega}}|\nabla u| / \sqrt{1+|\nabla u|^{2}}$,

$$
\begin{aligned}
\left|D_{1}\right| & \leqslant \int_{\Omega_{n}}|\nabla u||\nabla \chi| \frac{\left|\nabla u-\nabla u_{h}\right|\left(|\nabla u|+\left|\nabla u_{n}\right|\right)}{\sqrt{1+|\nabla u|^{2}} \sqrt{1+\left|\nabla u_{n}\right|^{2}}\left(\sqrt{1+|\nabla u|^{2}}+\sqrt{1+\left|\nabla u_{h}\right|^{2}}\right)} d x \\
& \leqslant \gamma \int_{\Omega_{n}} \frac{|\nabla \chi|\left|\nabla u-\nabla u_{h}\right|}{\sqrt{1+\left|\nabla u_{n}\right|^{2}}} d x \leqslant \gamma A\left(\int_{\Omega_{n}} \frac{|\nabla \chi|^{2}}{\sqrt{1+\left|\nabla u_{n}\right|^{2}}} d x\right)^{1 / 2} \\
& \leqslant \gamma A\left(A+\left|u-w_{h}\right|_{1,2, h}\right) .
\end{aligned}
$$

Thus 


$$
A^{2} \leqslant \gamma A\left(A+\left|u-w_{h}\right|_{1,2, h}\right)+A\left|u-w_{h}\right|_{1,2, h},
$$

so that, since $\gamma<1$,

$$
A \leqslant(1+\gamma)\left|u-w_{h}\right|_{1,2, h} /(1-\gamma)
$$

Now let $w_{h}$ agree with $u$ at the nodes. By a well-known estimate (cf., e.g., [3]), we then have

$$
\left|u-w_{h}\right|_{1,2, n} \leqslant C h|u|_{2,2},
$$

which completes the proof of the lemma.

As a consequence of Lemma 1 , we find

$$
\left\|\nabla u-\nabla u_{h}\right\|_{1, h} \leqslant\left(\int_{\Omega_{h}} \frac{\left|\nabla u-\nabla u_{h}\right|^{2}}{\sqrt{1+\left|\nabla u_{h}\right|^{2}}}\right)^{1 / 2}\left(\int_{\Omega_{h}} \sqrt{1+\left|\nabla u_{h}\right|^{2}} d x\right)^{1 / 2} \leqslant C h
$$

since, clearly, $\int_{\Omega_{h}} \sqrt{1+\left|\nabla u_{h}\right|^{2}} d x$ is bounded as a result of the minimizing property of $u_{n}$. In fact, Lemma 1 and (2.3) hold without the assumption that the edges of the triangles have length bounded below by $c h$. This assumption, however, will enter in the proof of the following lemma.

Lemma 2. Let $u \in W_{2}^{2}(\Omega) \cap W_{\infty}^{1}(\Omega)$. Then, there is a constant $C$ such that for any $0<h<1,\left\|\nabla u_{h}\right\|_{\infty, h} \leqslant C$.

Proof. By Lemma 1, we have, in particular, for any $T_{j} \subset \bar{\Omega}_{h}$,

$$
\left(\int_{T_{j}} \frac{\left|\nabla u-\nabla u_{n}\right|^{2}}{\sqrt{1+\left|\nabla u_{n}\right|^{2}}} d x\right)^{1 / 2} \leqslant C h
$$

so that

$$
\left(\int_{T_{j}} \frac{\left|\nabla u_{h}\right|^{2}}{\sqrt{1+\left|\nabla u_{h}\right|^{2}}} d x\right)^{1 / 2} \leqslant C h+C|u|_{1, \infty}\left(\int_{T_{j}} d x\right)^{1 / 2} \leqslant C h
$$

Since $\nabla u_{n}$ is constant on $T_{j}$, and the area of $T_{j}$ is bounded from below by a constant times $h^{2}$, it follows that

$$
\frac{\left|\nabla u_{n}\right|^{2}}{\sqrt{1+\left|\nabla u_{n}\right|^{2}}} \leqslant C \text { on } T_{j}
$$

and thus $\max _{\bar{\Omega}_{h}}\left|\nabla u_{h}\right| \leqslant C$, which proves the lemma.

Together with Lemma 1, this also completes the proof of Theorem 1.

3. Proof of Theorem 2. We shall now prove Theorem 2 using an adaptation of a duality argument employed previously for linear problems by, e.g., Nitsche [3] .

For technical reasons, we shall need to extend $u_{n}$ to a piecewise linear function defined on the polygonal domain $\widetilde{\Omega}_{h} \supset \Omega$ consisting of the union of the triangles which 
intersect $\bar{\Omega}$. To this end, we first extend $u \in W_{q}^{2}(\Omega)$ to a domain $\widetilde{\Omega}$ with $\widetilde{\Omega} \supset \Omega_{n}$ for $0<h<1$ in such a way that the extended $u$ belongs to $W_{q}^{2}(\widetilde{\Omega})$ (cf. [1]). We then extend $u_{n}$ to $\widetilde{\Omega}_{h}$ by setting $u_{n}$ equal to the linear function which interpolates the extended $u$ at the vertices of $T_{j}$ for each $T_{j} \subset \widetilde{\Omega}_{h} \backslash \Omega_{h}$. It is clear that, with $u_{h}$ extended in this fashion, the estimate of Theorem 1 holds, with $\Omega_{n}$ replaced by $\Omega$, i.e., $\left|u-u_{n}\right|_{1,2} \leqslant C h$.

We shall prove that, for any $p$ with $1 \leqslant p<2$, there is a constant $C$ such that $\left\|u-u_{h}\right\|_{p} \leqslant C h^{2}$, which implies Theorem 2 since $\Omega \supset \Omega_{h}$. By increasing $p$ or decreasing $q$, we may assume without loss of generality that $1 / p+1 / q=1$. It will, therefore, be sufficient to prove that there is a constant $C$ such that

$$
\left|\left(g, u-u_{n}\right)\right|=\left|\int_{\Omega} g\left(u-u_{n}\right) d x\right| \leqslant C h^{2}\|g\|_{q} \quad \text { for } g \in L_{q}(\Omega) .
$$

This will be accomplished by rewriting the left-hand side, interpreting $g$ as the righthand side of a certain linear elliptic equation.

For this purpose, let us start with the simple identity

$$
\int_{\Omega}\left[f_{, i}(\nabla u)-f_{, i}\left(\nabla u_{n}\right)\right] \chi_{, i} d x=\int_{\Omega} a_{i j}^{n}\left(u-u_{n}\right)_{, j} \chi_{, i} d x,
$$

where, for $x \in \Omega$,

$$
a_{i j}^{h}(x)=\int_{0}^{1} f_{, i j}\left(\nabla u_{h}(x)+s\left(\nabla u(x)-\nabla u_{h}(x)\right)\right) d s, \quad i, j=1,2 .
$$

Defining the bilinear form

$$
a_{n}(\chi, \psi)=\int_{\Omega} a_{i j}^{n} \chi, i,{ }_{, j} d x
$$

we notice that, by (2.1), (2.2) and (3.2), we have

$$
a_{n}\left(\chi, u-u_{n}\right)=0 \quad \text { for } \chi \in \stackrel{\circ}{S}_{n} .
$$

Since the coefficients of $a_{n}$ are discontinuous, it will be convenient to introduce also the bilinear form

$$
a(\chi, \psi)=\int_{\Omega} a_{i j} \chi_{, i} \psi_{, j} d x \quad \text { with } a_{i j}(x)=f_{, i j}(\nabla u(x)) .
$$

Since $u \in W_{q}^{2}(\Omega)$ and, in particular, $\nabla u$ is bounded, we find, using also (1.1), that the coefficients $a_{i j}$ satisfy the assumptions in the following lemma:

LEMmA 3. Assume that $a_{i j} \in W_{q}^{1}(\Omega)$ for some $q>2$ and that $a_{i j}(x) \xi_{i} \xi_{j}$ is uniformly elliptic in $\Omega$. Then, there exists a constant $C$ such that, for any $g \in L_{q}(\Omega)$, the Dirichlet problem

$$
-\left(a_{i j} v_{, i}\right)_{, j}=g \text { in } \Omega, v=0 \text { on } \Gamma,
$$

admits a unique solution $v \in W_{q}^{2}(\Omega)$ and

$$
\|v\|_{2, q} \leqslant C\|g\|_{q} .
$$


Proof. See [4, p. 203].

Multiplying (3.4) by $u-u_{h}$ and integrating by parts, we now find that $\left(g, u-u_{h}\right)$ can be rewritten in the following way:

$$
\begin{aligned}
\left(g, u-u_{h}\right) & =a\left(v, u-u_{h}\right)+\int_{\Gamma} v_{n}\left(u-u_{h}\right) d s \\
& =a_{h}\left(v, u-u_{h}\right)+\left(a-a_{h}\right)\left(v, u-u_{h}\right)+\int_{\Gamma} v_{n}\left(u-u_{h}\right) d s .
\end{aligned}
$$

Here $v_{n}=-n_{j} a_{i j} v_{, i}$, where $\left(n_{1}, n_{2}\right)$ is the outward normal to $\Gamma$. We shall prove that each of the three last terms is bounded by $C h^{2}\|g\|_{q}$, which will obviously prove the desired inequality (3.1).

To estimate the first term, let $v_{n} \in \stackrel{\circ}{S}_{n}$ interpolate $v$ on $\Omega$, so that $\left|v-v_{n}\right|_{1,2} \leqslant$ $C h|v|_{2,2}$. Since the coefficients of $a_{n}$ are bounded (cf. (1.1)), we thus find, by (3.3), (3.5) and Theorem 1, that

$$
\begin{aligned}
\left|a_{h}\left(v, u-u_{h}\right)\right| & =\left|a_{h}\left(v-v_{h}, u-u_{h}\right)\right| \leqslant C\left|v-v_{h}\right|_{1,2}\left|u-u_{h}\right|_{1,2} \\
& \leqslant C h^{2}|v|_{2,2} \leqslant C h^{2}\|g\|_{2} \leqslant C h^{2}\|g\|_{q} .
\end{aligned}
$$

Consider next the second term $\left(a-a_{n}\right)\left(v, u-u_{n}\right)$. Since the derivatives of the $f_{, i j}$ are bounded in $R^{2}$, we have

$$
\begin{aligned}
\left|a_{i j}-a_{i j}^{h}\right| & =\int_{0}^{1}\left[f_{, i j}(\nabla u)-f_{, i j}\left(\nabla u_{n}+s \nabla\left(u-u_{n}\right)\right)\right] d s \\
& \leqslant C\left|\nabla u-\nabla u_{n}\right| \quad \text { in } \Omega,
\end{aligned}
$$

so that

$$
\left\|a_{i j}-a_{i j}^{n}\right\|_{2} \leqslant C\left|u-u_{h}\right|_{1,2}, \quad i, j=1,2 .
$$

Further, by Sobolev's inequality and Lemma 3,

$$
|v|_{1, \infty} \leqslant C|v|_{2, q} \leqslant C\|g\|_{q} .
$$

Thus by Theorem 1,

$$
\begin{aligned}
\left|\left(a-a_{n}\right)\left(v, u-u_{n}\right)\right| & \leqslant C|v|_{1, \infty} \max _{i, j}\left\|a_{i j}-a_{i j}^{h}\right\|_{2}\left|u-u_{h}\right|_{1,2} \\
& \leqslant C h^{2}\|g\|_{q} .
\end{aligned}
$$

Finally, for the boundary term, we have by (3.6)

$$
\left|\int_{\Gamma} v_{n}\left(\varphi-u_{n}\right) d s\right| \leqslant C|v|_{1, \infty}\left\|\varphi-u_{n}\right\|_{1, \Gamma} \leqslant C\|g\|_{q}\left\|\varphi-u_{n}\right\|_{1, \Gamma}
$$

It is therefore sufficient to prove that

$$
\left\|\varphi-u_{h}\right\|_{1, \Gamma} \leqslant C h^{2}
$$


To see this, let $\varphi_{h}$ be the piecewise linear function of arc length $s$ defined on $\Gamma$ which agrees with $\varphi$ at the vertices on $\Gamma$. We then clearly have that $\left\|\varphi-\varphi_{h}\right\|_{1, \Gamma} \leqslant C h^{2}|\varphi|_{2,1, \Gamma}$, and therefore (3.7) will follow if we prove that $\left\|\varphi_{h}-u_{h}\right\|_{1, \Gamma} \leqslant C h^{2}$. To show this, we argue as follows: For any $\bar{P} \in \Gamma$, let $T_{j}$ be the triangle in $\widetilde{\Omega}_{h} \backslash \Omega_{h}$ such that $\bar{P} \in T_{j}$. Let $P_{1}$ and $P_{2}$ be the vertices of $T_{j}$ on $\Gamma$, let $s_{1}$ and $s_{2}$ be the arc lengths corresponding to $P_{1}$ and $P_{2}$, and assume that $\bar{P}$ corresponds to $s=s_{1}+\lambda\left(s_{2}-s_{1}\right)$ where $0 \leqslant \lambda \leqslant 1$.

Let now $P$ be the point on the chord $P_{1} P_{2}$ such that dist $\left(P, P_{1}\right)=\lambda \operatorname{dist}\left(P_{1}, P_{2}\right)$. Since we are interpolating linearly, we then have $\varphi_{h}(\bar{P})=u_{h}(P)$. It is easy to see that $\operatorname{dist}(\bar{P}, P) \leqslant C h^{2}$. Further, since $u_{n}$ is the interpolant of $u$ on $T_{j}$, we have that $\left|\nabla u_{n}\right|$ is bounded on $T_{j}$ and therefore

$$
\left|\varphi_{h}(\bar{P})-u_{h}(\bar{P})\right|=\left|u_{h}(P)-u_{h}(\bar{P})\right| \leqslant C h^{2} \quad \text { for } \bar{P} \in \Gamma,
$$

which implies that $\left\|\varphi_{n}-u_{n}\right\|_{1, \Gamma} \leqslant C h^{2}$. This completes the proof of Theorem 2 .

Mathematics Department

Chalmers Institute of Technology

Göteborg, Sweden

1. S. AGMON, Lectures on Elliptic Boundary Value Problems, Van Nostrand Math. Studies, No. 2, Van Nostrand, Princeton, N. J., 1965. MR 31 \#2504.

2. C. B. MORREY, JR., Multiple Integrals in the Calculus of Variations, Die Grundlehren der math. Wissenschaften, Band 130, Springer-Verlag, New York, 1966. MR 34 \#2380.

3. J. NITSCHE, "Lineare Spline-Funktionen und die Methoden von Ritz fur elliptische Randwertprobleme," Arch. Rational Mech. Anal., v. 36, 1970, pp. 348-355. MR 40 \#8250.

4. O. A. LADYŽENSKAYA (LADYZHENSKAJA) \& N. N. URAL'CEVA (URAL'TSEVA), Linear and Quasilinear Equations, Academic Press, New York, 1968. MR 39 \#5941. 